\title{
Comparison between Standard and Modified Denavit-Hartenberg Methods in Robotics Modelling
}

\author{
M. Granja, N. Chang, V. Granja, M. Duque, F. Llulluna \\ Escuela Politécnica Nacional \\ Ladrón de Guevara, E112-5 Quito, Ecuador \\ mario.granja@epn.edu.ec; nikolas.chang@epn.edu.ec; victoria.granja@epn.edu.ec; mauricio.duque@epn.edu.ec; \\ fredy.llulluna@epn.edu.ec
}

\begin{abstract}
In the present article, the matrix fundamental method of Denavit and Hartenberg standard and modified are faced, after the comparison has been made, it is demonstrated that in boot cases is similar the way how the links and joints are numerated. The links are numerated in ascending order starting from 0 fixed link to $\mathrm{n}$ the last thing, in the same way the joints are numerated from 1 going forward, following in the same case the cinematic chain, also the matrices product is used in the same sequence of the movements made for the mobile coordinates systems. The two methods has different forms to assign coordinates systems. In the standard D-H method the origin of the system I is along the axis of the articulation $\mathrm{i}+1$, and the sequence of movements is rotation and translation in $\mathrm{Z}$, after that a translation and rotation along $\mathrm{X}$ is made. In the modified $\mathrm{D}-\mathrm{H}$ method the origin of the systems $\mathrm{i}$ is along the joint $\mathrm{I}$, and the sequence of movements is rotation and translation along $\mathrm{X}$ and the rotation and translation along $\mathrm{Z}$.
\end{abstract}

Keywords: link, joint, articulated arm, degrees of freedom, Denavit-Hartenberg.

\section{Introduction}

The fundamental purpose of the present investigation is to compare the matrix method used in robotics to determinate the mechanism movement equations, constitute by open kinematic chains that has one fixed link, this mechanism is known in robotics as manipulator root, those articulated arms are essentials to obtain the equations that relation the coordinates of the last coordinates system of the final effector know as $o_{n} x_{n} y_{n} z_{n}$, with the coordinates of the fixed coordinates system $o_{0} x_{0} y_{0} z_{0}$. To this moment, only possibilities to improve the methods has been analyzed, or new methods has been presented to analyze mechanism through recent matrix analysis, but no a valid differentiation between boot methods due to the fact that each author choose the method that is more convenient or with whom he is familiarized.

The present work proposes the hypothesis: if the movements made to the mobile coordinates system are following the kinematic chain respect to the last coordinates system, then the matrix product must be done in the same sequence which allow us to obtain the movement equations. Known those movement equations the positions, velocity and acceleration could be obtained, in other words, the direct and inverse kinematic of the mechanism should be solved, to modelling those equations are required. In any case, the popularity of DH method has eclipsed so many alternatives in the field of basis robotics, possibly because of the less widely algebraic basis through the use of homogeneous transformation matrices to represent the changes of base without noticeable advantages over this, have had less acceptance that the method of Denavit and Hartenberg (Barrientos-Álvares, et al., 2012).

In section 2, some theory bases are described like how to assign the fixed coordinate system, and mobile coordinate systems and the importance of the sequence of movements made with the mobile system, these rotation matrices also show rotations and translation in the plane and space.

In section 3, the methods Denavit-Hartenberg standard and modified are used to assign the coordinate systems on the, Scara Robot forming the parameter tables and applying them to the matrix homogeneous transformation formula $A i$ (1), enabling to transform coordinates between two adjacent systems.

$$
{ }^{i-1} A_{i}=\left[\begin{array}{cccc}
\mathrm{c} \theta_{i} & -\mathrm{c} \propto_{i} * \mathrm{~s} \theta_{i} & \mathrm{~s} \propto_{i} * \mathrm{~s} \theta_{i} & a_{i} \mathrm{c} \theta_{i} \\
\mathrm{~s} \theta_{i} & \mathrm{c} \propto_{i} * \mathrm{c} \theta_{i} & -\mathrm{s} \propto_{i} * \mathrm{c} \theta_{i} & a_{i} \mathrm{~s} \theta_{i} \\
0 & \mathrm{~s} \propto_{i} & \mathrm{c} \propto_{i} & d_{i} \\
0 & 0 & 0 & 1
\end{array}\right]
$$


The matrix resulting Formula (2) to the arm is obtained by the expression:

$$
H=T_{n}^{0}=A_{1}\left(q_{1}\right) \ldots \ldots A_{n}\left(q_{n 1}\right)
$$

Section 4, conclusions are presented, it is supposed that the reader is familiarized with Denavit-Hartenberg method (Ollero. 2001), (Spong-Hutchinson, et al., 2004), (Barrientos et al. 2007), as well as term used to describe open kinematic chains (Fu, et al., 2002), (Craig, J. 2005).

\section{Theoretical Framework.}

The term mechanism is applied to the geometrical body combination that constitute a machine or part of a machine. Therefore, a mechanism can be defined as a combination of links and joints, so that they move with defined relative movement one respect to the other.

\subsection{Coordinates Systems, positions and orientation.}

The fundamental theory shown in this section would be necessary to determinate the direct and inverse kinematic, the representation used to determinate the model, is based in the equations used to translate or rotate coordinated systems from the fixed system $o_{0} x_{0} y_{0} z_{0}$ to the final effector of the robot in the system $o_{n} x_{n} y_{n} z_{n}$

\subsubsection{Rotation Matrix.}

\subsubsection{Plane}

The coordinates system $o_{0} x_{0} y_{0}$ is assigned to the fixed link, and the $o_{1} x_{1} y_{1}$ to the mobile link, at the first moment, the two origins of the systems are in the same position, and it is required to find the relation of the coordinate system $o_{0} x_{0} y_{0}$ as function of $x_{1} y_{1}$. Figure 1 represents how the 2 coordinated systems are related.

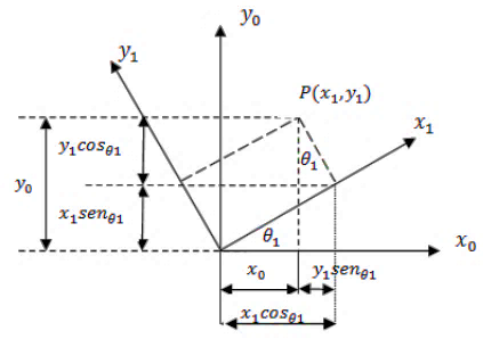

Fig. 1: Coordinate systems in a mobile and a fixed link.

Applying basic geometry to the representation in the figure 1, the equation referred to transform coordinates between the two systems is consequence of the axiom "The Everything is equal to the sum of its parts"; with this asseveration, we can obtain the equations and they could be added in a matrix arrangement along with the use of the equality one equals one.

$$
\left[\begin{array}{c}
x_{0} \\
y_{0} \\
1
\end{array}\right]=\left[\begin{array}{ccc}
\cos \theta_{1} & -\sin \theta_{1} & 0 \\
\sin \theta_{1} & \cos \theta_{1} & 0 \\
0 & 0 & 1
\end{array}\right]\left[\begin{array}{c}
x_{1} \\
y_{1} \\
1
\end{array}\right]
$$

\subsubsection{Spatial}

In spatial rotation, a point $\mathrm{P}$ and two systems with the same origin of coordinates must be considered, because the point will have two referred systems, the fixed system $o, x, y, z$ and the mobile system $o, u, v, w$; $\mathrm{P}$ could be represented by a position vector referred to the two coordinated systems. The unitary vectors of the systems are $\vec{\imath}_{x}, \vec{\jmath}_{y}, \vec{k}_{z}$, and $\vec{\imath}_{u}, \vec{\jmath}_{v}, \vec{k}_{w}$ respectively. The representation of the two coordinated systems and the position vector of the point $\mathrm{P}$ is shown in the figure 2 . With this representation, the point $P$ could be represented by the equations (4) or (5) 


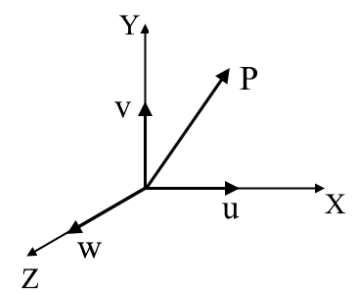

Fig. 2: Fixed and mobile coordinate systems with the position vector of $P$.

$$
\begin{gathered}
\vec{P}_{x, y, z}=P_{x} \vec{\imath}_{x}+P_{y} \vec{\jmath}_{y}+P_{z} \vec{k}_{z} \\
\vec{P}_{u, v, w}=P_{u} \vec{\imath}_{u}+P_{v} \vec{\jmath}_{v}+P_{w} \vec{k}_{w}
\end{gathered}
$$

Using the scalar product in the two position vectors (4) and the unitary vectors of (5), equation (6) is obtained.

$$
R=\left[\begin{array}{l}
p_{x} \\
p_{y} \\
p_{z}
\end{array}\right]=\left[\begin{array}{lll}
\vec{i}_{u} \cdot \vec{i}_{x} & \vec{j}_{v} \cdot \vec{i}_{x} & \vec{i}_{x} \\
\vec{j}_{v} \cdot \vec{i}_{y} & \vec{j}_{v} \cdot \vec{i}_{y} & \vec{j}_{v} \cdot \vec{i}_{y} \\
\vec{k}_{w} \cdot \vec{i}_{z} & \vec{k}_{w} \cdot \vec{i}_{z} & \vec{k}_{w} \cdot \vec{i}_{z}
\end{array}\right]\left[\begin{array}{c}
p_{u} \\
p_{v} \\
p_{w}
\end{array}\right]
$$

Using the matrix equation (6) and the figure 3 in which the rotation around axis $\mathrm{X}, \mathrm{Y}$ and $\mathrm{Z}$ are represented, the rotation matrices around those axes could be determinate and consequently the rotation matrices.
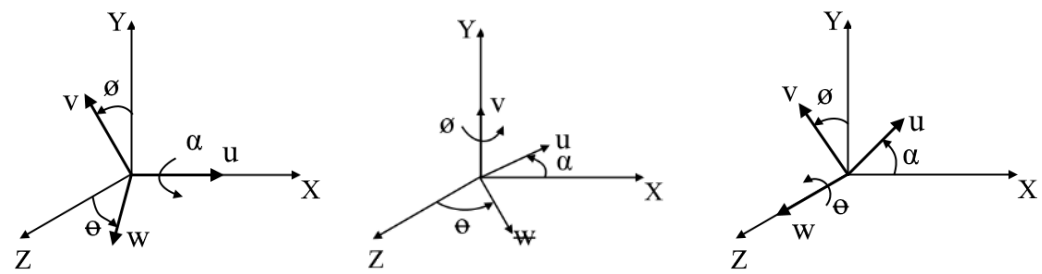

Fig. 3: Rotation of the system $\mathrm{p}, \mathrm{u}, \mathrm{v}, \mathrm{w}$ around $x, \propto, y, \phi z, \theta$.

$$
\begin{aligned}
R_{(x, \alpha)} & =\left[\begin{array}{cccc}
1 & 0 & 0 & 0 \\
0 & \cos \propto & -\operatorname{sen} \propto & 0 \\
0 & \operatorname{sen} \propto & \cos \propto & 0 \\
0 & 0 & 0 & 1
\end{array}\right] \\
R_{(y, \varnothing)}= & {\left[\begin{array}{cccc}
\cos \emptyset & 0 & \operatorname{sen} \emptyset & 0 \\
0 & 1 & 0 & 0 \\
-\operatorname{sen} \emptyset & 0 & \cos \emptyset & 0 \\
0 & 0 & 0 & 1
\end{array}\right] } \\
R_{(Z, \theta)} & =\left[\begin{array}{cccc}
c \theta & -\operatorname{sen} \theta & 0 & 0 \\
\operatorname{sen} \theta & \cos \theta & 0 & 0 \\
0 & 0 & 1 & 0 \\
0 & 0 & 0 & 1
\end{array}\right]
\end{aligned}
$$

\subsubsection{Translation Matrix}

\subsubsection{Plane}

Using figure 4, it is required to determinate the equations that make a relation between the coordinates of $P\left(x_{1}, y_{1}\right)$, with $P\left(x_{0}, y_{0}\right)$, after a translation of the mobile system $\Delta x$ along the axis $x_{1}$ and another translation $\Delta y$ along the axis $y_{1}$. 


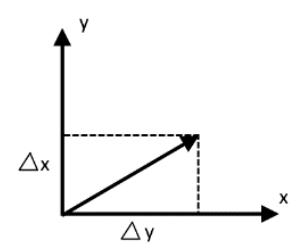

Fig. 4: Assignment of the coordinated systems in planar translation.

The equations that relation these two systems, as the first ones, could be added the identity $1=1$ to normalize the equations in one matrix $3 \times 3$, as shown in equation (10).

$$
\left[\begin{array}{c}
x_{0} \\
y_{0} \\
1
\end{array}\right]=\left[\begin{array}{ccc}
1 & 0 & \Delta x \\
0 & 1 & \Delta y \\
0 & 0 & 1
\end{array}\right]\left[\begin{array}{c}
x_{1} \\
y_{1} \\
1
\end{array}\right] \quad T=\left[\begin{array}{ccc}
1 & 0 & \Delta x \\
0 & 1 & \Delta y \\
0 & 0 & 1
\end{array}\right]
$$

\subsubsection{Spatial}

In a spatial translation, a point $\mathrm{P}$ and a fixed coordinated system $o, x, y, z$ and the mobile system $o, u, v, w$ that has been translated $\Delta x$ along the axis $x_{1}$, another translation $\Delta y$ along the axis $y_{1}$, and one translation $\Delta z$ along the axis $z_{1}$. The coordinates of the point $\mathrm{P}$ are known, and it moves with the mobile system. To determinate the equations to relation the system $o, x, y, z$ as function of $o, u, v, w$, the figure 5 will be used and considering the identity $1=1$ we can obtain equations that relation the systems only by geometrical relations.

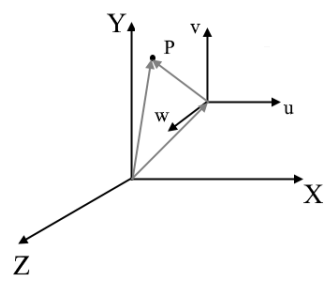

Fig. 5: assignation of the coordinated systems in spatial translation.

$$
\begin{gathered}
p_{x}=\vec{P}_{u}+\Delta x \\
p_{y}=\vec{P}_{v}+\Delta y \\
p_{z}=\vec{P}_{w}+\Delta z \\
1=1
\end{gathered}
$$

Applying the matrix method to the equations obtained, the matrix representation of the transformation of coordinates is obtained in equation (14)

$$
\left[\begin{array}{c}
p_{x} \\
p_{y} \\
p_{z} \\
1
\end{array}\right]=\left[\begin{array}{cccc}
1 & 0 & 0 & 0 \\
0 & \cos \propto & -\operatorname{sen} \propto & 0 \\
0 & \operatorname{sen} \propto & \cos \propto & 0 \\
0 & 0 & 0 & 1
\end{array}\right]\left[\begin{array}{c}
p_{u} \\
p_{v} \\
p_{w} \\
1
\end{array}\right] \quad T=\left[\begin{array}{cccc}
1 & 0 & 0 & 0 \\
0 & \cos \propto & -\operatorname{sen} \propto & 0 \\
0 & \operatorname{sen} \propto & \cos \propto & 0 \\
0 & 0 & 0 & 1
\end{array}\right]
$$

\section{Direct Kinematics.}

To make a simple comparison between the methods and validate its results, before using it in the Scara Robot, the comparison will be applied in a two dimensional case. 


\subsection{Two dimensional Case.}

\subsubsection{Standard Denavit-Hartenberg method (DHs).}

Mechanism DOF is considered, it is shown in the figure 6, where initially the two coordinated systems, the fixed $o_{0} x_{0} y_{0}$ and mobile $o_{n} x_{n} y_{n}$ are overlapped, following the DHs method, first with the mobile system we rotate a $\theta 1$ angle around $Z$ axis, after a translation along the new axis $\mathrm{x}$ with the value $a l$, the final system located in the joint 1 would be called $o_{1} x_{1} y_{1}$; In the same way that the other link, first we rotate an angle $\theta 2$, after a translation along the new axis $\mathrm{x}$ with the value of $a 2$, the final system located in the joint 2 would be called $o_{2} x_{2} y_{2}$; as seen all the movements were made with respect to the axis of the mobile coordinate system.

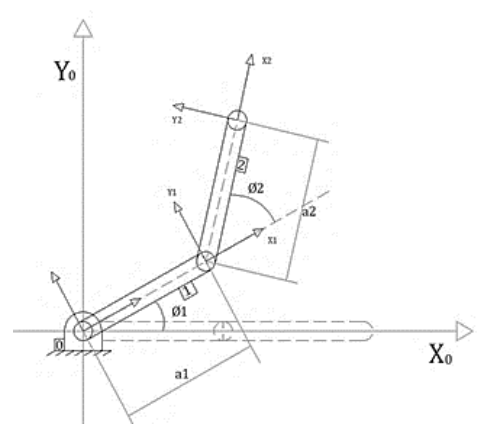

Fig. 6: assignation of the coordinated systems over the link i, DHs.

The equations of coordinate transformation between the system 2 and 0 , are in matrix notation, it is find multiplying the matrices in the same sequence of the movements, rotations and translation in each part of the link, like it shown next. Movements over link 1: the equations of coordinate's transformations between the fixed systems $o_{0} x_{0} y_{0}$, the system o, $\mathrm{x}$, $\mathrm{y}$, after the rotation is represented by:

$$
\begin{gathered}
{\left[\begin{array}{c}
x_{0} \\
y_{0} \\
1
\end{array}\right]=\left[\begin{array}{ccc}
\cos \theta_{1} & -\sin \theta_{1} & 0 \\
\sin \theta_{1} & \cos \theta_{1} & 0 \\
0 & 0 & 1
\end{array}\right]\left[\begin{array}{ccc}
1 & 0 & \Delta x 1 \\
0 & 1 & 0 \\
0 & 0 & 1
\end{array}\right]\left[\begin{array}{c}
x_{1} \\
y_{1} \\
1
\end{array}\right]=\left[\begin{array}{ccc}
\cos \theta_{1} & -\sin \theta_{1} & \Delta x 1 \cos \theta_{1} \\
\sin \theta_{1} & \cos \theta_{1} & \Delta x 1 \sin \theta_{1} \\
0 & 0 & 1
\end{array}\right]\left[\begin{array}{c}
x_{1} \\
y_{1} \\
1
\end{array}\right]} \\
A_{1}=\left[\begin{array}{ccc}
\cos \theta_{1} & -\sin \theta_{1} & \Delta x 1 \cos \theta_{1} \\
\sin \theta_{1} & \cos \theta_{1} & \Delta x 1 \sin \theta_{1} \\
0 & 0 & 1
\end{array}\right] \quad\left[\begin{array}{c}
x_{0} \\
y_{0} \\
1
\end{array}\right]=A_{1}\left[\begin{array}{c}
x_{1} \\
y_{1} \\
1
\end{array}\right]
\end{gathered}
$$

Movements over link 2: Being the same base movements, the equation obtained to transform coordinate systems from link 1 to the link 2 using matrix notation we have:

$$
\begin{gathered}
{\left[\begin{array}{c}
x_{1} \\
y_{1} \\
1
\end{array}\right]=\left[\begin{array}{ccc}
\cos \theta_{2} & -\sin \theta_{2} & 0 \\
\sin \theta_{2} & \cos \theta_{2} & 0 \\
0 & 0 & 1
\end{array}\right]\left[\begin{array}{ccc}
1 & 0 & \Delta x 2 \\
0 & 1 & 0 \\
0 & 0 & 1
\end{array}\right]\left[\begin{array}{c}
x_{2} \\
y_{2} \\
1
\end{array}\right]=\left[\begin{array}{ccc}
\cos \theta_{2} & -\sin \theta_{2} & \Delta x 2 \cos \theta_{2} \\
\sin \theta_{2} & \cos \theta_{2} & \Delta x 2 \sin \theta_{2} \\
0 & 0 & 1
\end{array}\right]\left[\begin{array}{c}
x_{2} \\
y_{2} \\
1
\end{array}\right]} \\
A_{2}=\left[\begin{array}{ccc}
\cos \theta_{2} & -\sin \theta_{2} & \Delta x 2 \cos \theta_{2} \\
\sin \theta_{2} & \cos \theta_{2} & \Delta x 2 \sin \theta_{2} \\
0 & 0 & 1
\end{array}\right]\left[\begin{array}{c}
x_{0} \\
y_{0} \\
1
\end{array}\right]=A_{2}\left[\begin{array}{c}
x_{1} \\
y_{1} \\
1
\end{array}\right]
\end{gathered}
$$

Matrix $\mathrm{T}$ of the arm: Replacing the equations (17) and (18), to obtain the matrix of transformation of coordinates from the fixed coordinate system 0 , to the final system 2, this matrix is known as the arm matrix. Also is important to clarify that in most of the robotics books, to easily write the large trigonometrical expressions the use of sub index to represent the articular variables, in that $\operatorname{case} \cos \theta_{1}$ is equivalent to write $c_{1}$.

\subsubsection{Modified Denavit-Hartenberg method (DHm)}

Using the same mechanisms figure 6, the fixed system has the coordinate system called o_1 $\mathrm{x}_{-} 1 \mathrm{y} \_1$, first the mobile is rotated around $\mathrm{Z}$ an angle $\theta_{-} 1$, after that a translation around the new axis $\mathrm{x}$ is made with a value $\Delta \mathrm{x} 1$, to this rotated and 
translated coordinated system is assigned the name o_2 $\mathrm{x} \_2 \mathrm{y} \_2$, in which the subscripts 1 shown that the movements were around the joint 1; as same as the joint 1 , over the link 2 a rotation $\theta \_2$ is made around the $\mathrm{Z}$ axis, after that a translation is made with the new $\mathrm{x}$ axis with value $\Delta \mathrm{x} 2$, to this rotated and translated coordinated system is assigned the name o_3 $\mathrm{x}_{-} 3$ y_3.

$$
\begin{gathered}
{\left[\begin{array}{c}
x_{0} \\
y_{0} \\
1
\end{array}\right]=A_{1} A_{2}\left[\begin{array}{c}
x_{2} \\
y_{2} \\
1
\end{array}\right]} \\
T_{2}^{0}=A_{1} A_{2}=\left[\begin{array}{ccc}
c_{12} & -s_{12} & \Delta x 2 c_{12}+\Delta x 1 c_{1} \\
s_{12} & c_{12} & \Delta x 2 s_{12}+\Delta x 1 c_{1} \\
0 & 0 & 1
\end{array}\right] \\
{\left[\begin{array}{c}
x_{0} \\
y_{0} \\
1
\end{array}\right]=\left[\begin{array}{ccc}
c_{12} & -s_{12} & \Delta x 2 c_{12}+\Delta x 1 c_{1} \\
s_{12} & c_{12} & \Delta x 2 s_{12}+\Delta x 1 c_{1} \\
0 & 0 & 1
\end{array}\right]\left[\begin{array}{c}
x_{2} \\
y_{2} \\
1
\end{array}\right]}
\end{gathered}
$$

Where: $c_{12}=\cos \left(\theta_{1}+\theta_{2}\right) ; s_{12}=\sin \left(\theta_{1}+\theta_{2}\right) ; c_{1}=\cos \left(\theta_{1}\right) ; s_{1}=\sin \left(\theta_{1}\right)$

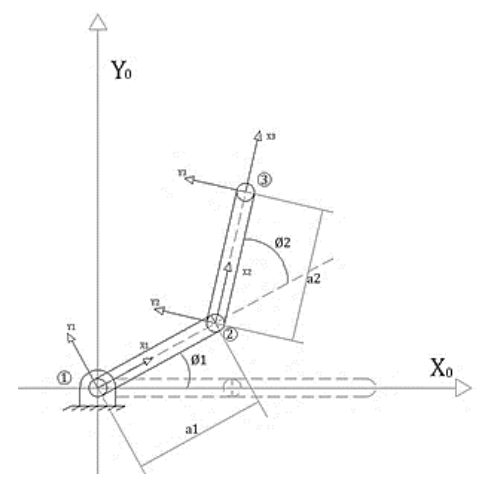

Fig. 7: assignation of the coordinated systems over the joints i, DH.

The equations of coordinate transformation between the system 3 and 0 in matrix notation is found multiplying the matrices in the same sequence of the movements (matrix post-multiplication); it's worth say that in this method the movements occur first in the $\mathrm{X}$ axis, being different of the standard where the movements begin in the $\mathrm{Z}$ axis.

Joint 1: The matrix obtained is the equation of coordinates transformation between the systems $o_{0} x_{0} y_{0}$ and the system $o_{1} x_{1} y_{1}$.

$$
\begin{gathered}
{\left[\begin{array}{c}
x_{0} \\
y_{0} \\
1
\end{array}\right]=R_{z, \theta 1}\left[\begin{array}{c}
x_{1} \\
y_{1} \\
1
\end{array}\right]=A_{1}\left[\begin{array}{c}
x_{1} \\
y_{1} \\
1
\end{array}\right] \quad A_{1}=\left[\begin{array}{ccc}
\cos \theta_{1} & -\sin \theta_{1} & 0 \\
\sin \theta_{1} & \cos \theta_{1} & 0 \\
0 & 0 & 1
\end{array}\right]} \\
{\left[\begin{array}{c}
x_{0} \\
y_{0} \\
1
\end{array}\right]=\left[\begin{array}{ccc}
\cos \theta_{1} & -\sin \theta_{1} & 0 \\
\sin \theta_{1} & \cos \theta_{1} & 0 \\
0 & 0 & 1
\end{array}\right]\left[\begin{array}{c}
x_{1} \\
y_{1} \\
1
\end{array}\right]}
\end{gathered}
$$

Joint 2: The matrix obtained is the equation of coordinates transformation between the systems $o_{1} x_{1} y_{1}$ and the system $o_{2} x_{2} y_{2}$, in this system 2 movements occurred, a translation along the $\mathrm{x}$ axis and a rotation around de $\mathrm{Z}$ axis.

$$
\left[\begin{array}{c}
x_{1} \\
y_{1} \\
1
\end{array}\right]=T_{x, \Delta x 1} R_{z, \theta 2}\left[\begin{array}{c}
x_{2} \\
y_{2} \\
1
\end{array}\right]=A_{2}\left[\begin{array}{c}
x_{2} \\
y_{2} \\
1
\end{array}\right]
$$




$$
\begin{gathered}
A_{2}=\left[\begin{array}{ccc}
1 & 0 & \Delta x 1 \\
0 & 1 & 0 \\
0 & 0 & 1
\end{array}\right]\left[\begin{array}{ccc}
\cos \theta_{2} & -\sin \theta_{2} & 0 \\
\sin \theta_{2} & \cos \theta_{2} & 0 \\
0 & 0 & 1
\end{array}\right]=\left[\begin{array}{ccc}
\cos \theta_{2} & -\sin \theta_{2} & \Delta x 1 \\
\sin \theta_{2} & \cos \theta_{2} & 0 \\
0 & 0 & 1
\end{array}\right] \\
{\left[\begin{array}{c}
x_{1} \\
y_{1} \\
1
\end{array}\right]=\left[\begin{array}{ccc}
\cos \theta_{2} & -\sin \theta_{2} & \Delta x 1 \\
\sin \theta_{2} & \cos \theta_{2} & 0 \\
0 & 0 & 1
\end{array}\right]\left[\begin{array}{c}
x_{2} \\
y_{2} \\
1
\end{array}\right]}
\end{gathered}
$$

Joint 3: The matrix obtained is the equation of coordinates transformation between the systems $o_{2} x_{2} y_{2}$ and the system $o_{3} x_{3} y_{3}$, in this system just a translation occurred along the $\mathrm{X}$ axis.

$$
\begin{gathered}
{\left[\begin{array}{c}
x_{2} \\
y_{2} \\
1
\end{array}\right]=T_{x, \Delta x 1}\left[\begin{array}{c}
x_{3} \\
y_{3} \\
1
\end{array}\right]=A_{3}\left[\begin{array}{c}
x_{3} \\
y_{3} \\
1
\end{array}\right] \quad A_{3}=\left[\begin{array}{ccc}
1 & 0 & \Delta x 2 \\
0 & 1 & 0 \\
0 & 0 & 1
\end{array}\right]} \\
{\left[\begin{array}{c}
x_{2} \\
y_{2} \\
1
\end{array}\right]=\left[\begin{array}{ccc}
1 & 0 & \Delta x 2 \\
0 & 1 & 0 \\
0 & 0 & 1
\end{array}\right]\left[\begin{array}{c}
x_{3} \\
y_{3} \\
1
\end{array}\right]}
\end{gathered}
$$

Matrix $\mathrm{T}$ of the arm: Replacing the equations (36), (38) and (40), it is obtained the matrix of transformation of coordinates from the fixed coordinate system 0 , to the final system 3 , this matrix is known as the arm matrix.

$$
\begin{gathered}
{\left[\begin{array}{c}
x_{0} \\
y_{0} \\
1
\end{array}\right]=A_{1} A_{2} A_{3}\left[\begin{array}{c}
x_{3} \\
y_{3} \\
1
\end{array}\right]} \\
T_{3}^{0}=A_{1} A_{2} A_{3}=\left[\begin{array}{ccc}
c_{12} & -s_{12} & \Delta x 2 c_{12}+\Delta x 1 c_{1} \\
s_{12} & c_{12} & \Delta x 2 s_{12}+\Delta x 1 c_{1} \\
0 & 0 & 1
\end{array}\right] \\
{\left[\begin{array}{c}
x_{0} \\
y_{0} \\
1
\end{array}\right]=\left[\begin{array}{ccc}
c_{12} & -s_{12} & \Delta x 2 c_{12}+\Delta x 1 c_{1} \\
s_{12} & c_{12} & \Delta x 2 s_{12}+\Delta x 1 c_{1} \\
0 & 0 & 1
\end{array}\right]\left[\begin{array}{c}
x_{3} \\
y_{3} \\
1
\end{array}\right]}
\end{gathered}
$$

\subsubsection{Continuous General Movement (MGC).}

The mechanism in the figure 8 has two coordinates systems, the fixed $o_{0} x_{0} y_{0}$, and the mobile system $\mathrm{o}, \mathrm{x}, \mathrm{y}$. Following the steps of the MGC method, we do not have to consider any joint or link, we are going to move through the mechanism following the kinematic chain in the order explained next:

1. Rotation around $\mathrm{Z}$ an angle $\theta_{1}$

2. Translation along the new $\mathrm{X}$ axis a $\Delta x 1$ value

3. Rotation around $\mathrm{Z}$ an angle $\theta_{2}$

4. Translation along the new $\mathrm{X}$ axis a $\Delta x 2$ value

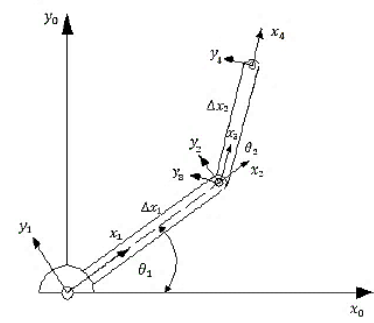

Fig. 8: Coordinates system assigned by the sequence of movements. 
Following the method, the next step is to obtain the matrices to transform every movement made during the way to reach the final of the mechanism, the equations of contiguous systems are:

$$
\begin{aligned}
& {\left[\begin{array}{c}
x_{0} \\
y_{0} \\
1
\end{array}\right]=R_{z, \theta 1}\left[\begin{array}{c}
x_{1} \\
y_{1} \\
1
\end{array}\right] ;\left[\begin{array}{c}
x_{1} \\
y_{1} \\
1
\end{array}\right]=T_{x, \Delta x 1}\left[\begin{array}{c}
x_{2} \\
y_{2} \\
1
\end{array}\right] ;\left[\begin{array}{c}
x_{2} \\
y_{2} \\
1
\end{array}\right]=R_{z, \theta 2}\left[\begin{array}{c}
x_{3} \\
y_{3} \\
1
\end{array}\right] ;\left[\begin{array}{c}
x_{3} \\
y_{3} \\
1
\end{array}\right]=T_{x, \Delta x 2}\left[\begin{array}{c}
x_{4} \\
y_{4} \\
1
\end{array}\right]} \\
& {\left[\begin{array}{c}
x_{0} \\
y_{0} \\
1
\end{array}\right]=R_{z, \theta 1} \cdot T_{x, \Delta x 1} \cdot R_{z, \theta 2} \cdot T_{x, \Delta x 2}\left[\begin{array}{c}
x_{4} \\
y_{4} \\
1
\end{array}\right]} \\
& T_{4}^{0}=R_{z, \theta 1} \cdot T_{x, \Delta x 1} \cdot R_{z, \theta 2} \cdot T_{x, \Delta x 2} \\
& T_{4}^{0}=\left[\begin{array}{ccc}
\cos \theta_{1} & -\sin \theta_{1} & 0 \\
\sin \theta_{1} & \cos \theta_{1} & 0 \\
0 & 0 & 1
\end{array}\right]\left[\begin{array}{ccc}
1 & 0 & \Delta x 1 \\
0 & 1 & 0 \\
0 & 0 & 1
\end{array}\right] \cdot\left[\begin{array}{ccc}
\cos \theta_{2} & -\sin \theta_{2} & 0 \\
\sin \theta_{2} & \cos \theta_{2} & 0 \\
0 & 0 & 1
\end{array}\right]\left[\begin{array}{ccc}
1 & 0 & \Delta x 2 \\
0 & 1 & 0 \\
0 & 0 & 1
\end{array}\right] \\
& T_{4}^{0}=\left[\begin{array}{ccc}
c_{12} & -s_{12} & \Delta x 2 c_{12}+\Delta x 1 c_{1} \\
s_{12} & c_{12} & \Delta x 2 s_{12}+\Delta x 1 c_{1} \\
0 & 0 & 1
\end{array}\right]
\end{aligned}
$$

As the reader can see, the results in MGC are the same and easily to understand in planar open mechanisms and easier to achieve with less iterations of the work and without any assumption, just following the kinematic chain from the bottom to the final.

\subsection{Comparison between the methods in the Scara Robot.}

\subsubsection{Standard}

Denavit and Hartenberg organized the sequence of movements in groups of four to reach the link $i$, from the coordinates system $(i-1)$ to the next coordinates system $i$. This agreement is used in the figure 9 . All the movements made to the mobile coordinates system are presented formally in groups of four, respect to each link i, in table 1 .

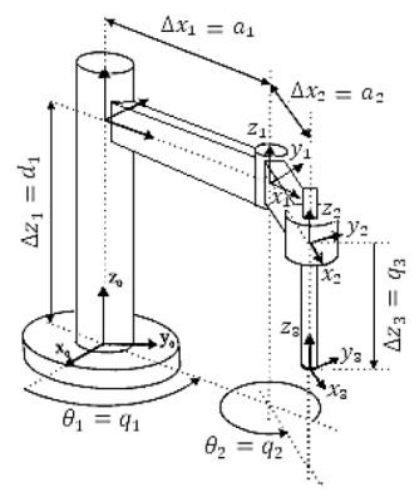

Fig. 9. Coordinates systems D-H standard.

Table 1. Parameters D-H Standard Table.

\begin{tabular}{|l|c|c|c|c|}
\hline Link i & $\begin{array}{c}\theta_{i} \\
\text { Rot z }\end{array}$ & $\begin{array}{c}d_{i} \\
\text { Disp z }\end{array}$ & $\begin{array}{c}a_{i} \\
\text { Disp x }\end{array}$ & $\begin{array}{c}\alpha_{i} \\
\operatorname{Rot} \mathrm{x}\end{array}$ \\
\hline 1 & $\theta_{1}{ }^{*}$ & $d_{1}$ & $a_{1}$ & 0 \\
\hline 2 & $\theta_{2}{ }^{*}$ & 0 & $a_{2}$ & 0 \\
\hline 3 & 0 & $-q_{3}$ & 0 & 0 \\
\hline
\end{tabular}

Rot: Rotation around, Disp: Displacement along 
The parameters of each row correspond to the sequence of the movements made by the mobile coordinates system in the link $I$, those parameters and variables (marked with an asterisk) are replaced in the coordinates transformation matrix $A i$.

$$
H=\left[\begin{array}{cccc}
\cos \left(\theta_{1}+\theta_{2}\right) & -\operatorname{sen}\left(\theta_{1}+\theta_{2}\right) & 0 & a_{2} \cos \left(\theta_{1}+\theta_{2}\right)+a_{2} \cos \left(\theta_{1}\right) \\
\operatorname{sen}\left(\theta_{1}+\theta_{2}\right) & \cos \left(\theta_{1}+\theta_{2}\right) & 0 & a_{2} \operatorname{sen}\left(\theta_{1}+\theta_{2}\right)+a_{2} \operatorname{sen}\left(\theta_{1}\right) \\
0 & 0 & 1 & d_{1}-q_{3} \\
0 & 0 & 0 & 1
\end{array}\right]
$$

\subsubsection{Modified}

To apply this method, the sequence of movements is grouped in 4 as the standard method, the main difference is how these groups are formed beginning from the coordinates system $s\{i-1\}$ to the system $s\{i\}$, the assignation is shown in figure $\mathrm{y}$, and those movements are formally presented in table $\mathrm{y}$ according to the agreement.

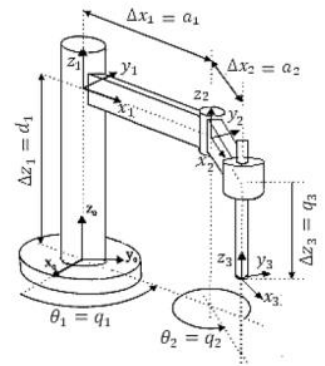

Fig. 10. Coordinates systems D-H modified.

Table 2. Parameters D-H Modified Table.

\begin{tabular}{|l|c|c|c|c|}
\hline Joint i & $\begin{array}{c}\alpha_{i-1} \\
\text { Rot x }\end{array}$ & $\begin{array}{c}a_{i-1} \\
\text { Disp x }\end{array}$ & $\begin{array}{c}\theta_{i} \\
\text { Rot z }\end{array}$ & $\begin{array}{c}d_{i} \\
\text { Disp z }\end{array}$ \\
\hline 1 & 0 & 0 & $\theta_{1}{ }^{*}$ & $d_{1}$ \\
\hline 2 & 0 & $a_{1}$ & $\theta_{2}{ }^{*}$ & 0 \\
\hline 3 & 0 & $a_{2}$ & 0 & $-q_{3}$ \\
\hline
\end{tabular}

Rot: Rotation around, Disp: Displacement along.

The parameters in each row of the table correspond to the sequence of movements made to the coordinate system to pass the joint $i$, the main difference is the sequence of movements, that are made first along the $\mathrm{X}$ axis and later along the $\mathrm{Z}$ axis, those parameter and variables (mark with asterisk) are replaced in the coordinates transformation matrix formula Ai, also known as Denavit-Hartenberg modified.

$$
\begin{gathered}
A_{i}=R_{x_{i-1}, \alpha_{i-1}} \cdot T_{x_{i-1}, \alpha_{i-1}} \cdot R_{z, \theta i} \cdot T_{z, \mathrm{~d} i} \\
A_{i}=\left[\begin{array}{cccc}
\cos \left(\theta_{i}\right) & -\sin \left(\theta_{i}\right) & 0 & a_{i-1} \\
\sin \left(\theta_{i}\right) \cos \left(\alpha_{i-1}\right) & \cos \left(\theta_{i}\right) \cos \left(\alpha_{i-1}\right) & -\sin \left(\alpha_{i-1}\right) & -d_{i} \sin \left(\alpha_{i-1}\right) \\
\sin \left(\theta_{i}\right) \cos \left(\alpha_{i-1}\right) & \cos \left(\theta_{i}\right) \sin \left(\alpha_{i-1}\right) & \cos \left(\alpha_{i-1}\right) & d_{i} \cos \left(\alpha_{i-1}\right) \\
0 & 0 & 0 & 1
\end{array}\right]
\end{gathered}
$$

Then applying the concept of the homogeneous transformation matrix, $\mathrm{H}$ is obtained:

$$
H=T_{3}^{0}=A_{1}^{0} \cdot A_{2}^{1} \cdot A_{3}^{2}
$$




$$
H=\left[\begin{array}{cccc}
\cos \left(\theta_{1}+\theta_{2}\right) & -\operatorname{sen}\left(\theta_{1}+\theta_{2}\right) & 0 & a_{2} \cos \left(\theta_{1}+\theta_{2}\right)+a_{2} \cos \left(\theta_{1}\right) \\
\operatorname{sen}\left(\theta_{1}+\theta_{2}\right) & \cos \left(\theta_{1}+\theta_{2}\right) & 0 & a_{2} \operatorname{sen}\left(\theta_{1}+\theta_{2}\right)+a_{2} \operatorname{sen}\left(\theta_{1}\right) \\
0 & 0 & 1 & d_{1}-q_{3} \\
0 & 0 & 0 & 1
\end{array}\right]
$$

Obtaining the same exact result by using the two methods.

\section{Conclusions}

The present investigations confirm the principle of post and pre matrix multiplication that is the fundamental base of matrix methods in robotics.

Post multiplication principle said: if the movements of the coordinates system are respect to the axis of the last mobile coordinate system, then the matrix product should be done in the same sequence of those movements.

Pre multiplication principle said: if the movements of the coordinates system are respect to the fixed link, then the matrix product should be done in reverse sequence of those movements.

The terms screw $\mathrm{X}$ or $\mathrm{Z}$ means that it has two movements, one of rotation and other of translation, going forward along the corresponding axis.

The Denavit-Hartenberg method standard assign coordinates systems to the link i over the axis line that join the link and the articulation $\mathrm{i}+1$, while the modified method assigns coordinates systems to the joint $\mathrm{i}$ over the line that join the articulation with the link $i+1$.

\section{References}

[1] J. Denavit, R. S. Hartenberg, "A kinematic notation for lower-pair mechanisms based on matrices," ASME Journal of Applied Mechanisms, vol. 23, pp. 215-221, 1955.

[2] R. Balasubramanian, "The Denavit Hartenberg Convention," USA: Robotics Insitute Carnegie Mellon University, 2011.

[3] A. Barrientos, L. Peñin, C. Balaguer, R. Aracil, Fundamentos de Robótica, 2nd Edition, McGraw-Hill, 2007.

[4] J. Craig, "Introduction to robotics mechanics and control," 3rd edition, USA: PEARSON Prentice Hall, 2005.

[5] K. Fu, R. Gonzalez, C. Lee, "Robótica: Control, Detección, Visión e Inteligencia,” España: Ed. Mc Graw Hill.

[6] A. Jochheim, M. Gerke, A. Bischoff, "Modeling and Simulation of Kinematic Systems," Alemania: University of Hagen, 1999.

[7] H. Mabie, Mechanism and Dynamics of Machinery. USA: John Wiley \& Son, 1987.

[8] A. Ollero, Robótica: Manipuladores y robots móviles. 1st Edition, MARCOMBO, 2001.

[9] J. Shigley and Jr. J Uicker, Teoría De Maquinas Y Mecanismos. Mexico: Mc Graw Hill, 1988.

[10] M. Spong, S. Hutchinson, M. Vidyasagar, "Robot Dynamics and Control," USA: ED. John Wiley \& Sons, Inc, 2004.

[11] P.I. Corke, “A Robotics Toolbox for MATLAB,” Robotics \& Automation Magazine, IEEE, vol.3(1), pp. 24-32, 1996.

[12] M. Granja, "Comparación entre el Método Denavit-Hartenberg Estándar y Modificado,” ECUADOR: EPN, 2014. 